\title{
Estudio de la relación polen-vegetación actual en el Norte de Chile, en el transecto Pozo Almonte-Salar de Huasco (20¹5'S/6906'O)
}

\section{Study of the current relationship between pollen and vegetation in the North of Chile, in the Pozo Almonte - Salar de Huasco transect $\left(20^{\circ} 15^{\prime} \mathrm{S} / 69^{\circ} 06^{\prime} \mathrm{W}\right)$}

\author{
Kathy Collao-Alvarado ${ }^{*}$, Antonio Maldonado ${ }^{2,3}$, Leticia González ${ }^{2}$, Ana Sandoval ${ }^{2}$, María Eugenia \\ de Porras ${ }^{2}$, Andrés Zamora ${ }^{2} \&$ Gina Arancio $^{4}$ \\ ${ }^{1}$ Facultad de Ciencias, Universidad de Chile, Las Palmeras 3425, 7800024 Santiago, Chile. \\ ${ }^{2}$ Centro de Estudios Avanzados en Zonas Áridas, Universidad de La Serena, Colina El Pino s/n, La Serena, Chile. \\ ${ }^{3}$ Universidad Católica del Norte, Larrondo 1281, Coquimbo, Chile. \\ ${ }^{4}$ Departamento de Biología, Facultad de Ciencias, Universidad de La Serena, La Serena, Chile. \\ *k.collao.a@gmail.com
}

\section{RESUMEN}

La diversidad vegetal de la zona andina del Norte Grande de Chile ha sido estudiada desde diversos puntos de vista (florísticos, fisonómicos, etnobotánicos, conservacionistas, etc.); sin embargo, hasta ahora no se ha estudiado desde un punto de vista palinológico. Así, este trabajo tiene como objetivo aportar al conocimiento de la vegetación y su señal polínica en los distintos pisos altitudinales del Norte Grande de Chile, aportando además a la identificación de los principales tipos polínicos presentes. Este estudio presenta la relación entre lluvia de polen y vegetación actual en un transecto altitudinal entre Pozo Almonte y el Salar de Huasco, en el norte de Chile, junto con fotografías y descripciones de 12 morfotipos polínicos representativos de la zona. Los resultados de los muestreos de vegetación corroboran las tendencias descritas en trabajos previos identificándose los principales pisos de vegetación descritos anteriormente (piso prepuneño, puneño, altoandino y subnival). De igual manera los resultados de la lluvia de polen muestran una clara relación con los ensambles de vegetación que la produce, siendo posible diferenciar cada uno de los pisos de vegetación a partir de su señal polínica. Así, los resultados pueden ser de gran interés para la interpretación de registros polínicos fósiles o para estudios de carácter ecológico.

Palabras clave: Palinología, lluvia de polen, pisos altitudinales, diversidad vegetal.

\begin{abstract}
The plant diversity found in the Andean region of the Norte Grande area in Chile has been studied from different points of view (floral, physiognomy, ethnobotanical, conservationists, etc.). Nevertheless, there has not been any research done from a palynological point of view until now. Thus, this investigation's objective is to contribute knowledge about the vegetation and its pollen signal in the different altitudinal tiers of the Norte Grande area in Chile, and to help identify the main pollen types present here. This research shows the relationship between pollen rain and current vegetation in the Pozo Almonte and Salar de Huasco altitudinal transect in the north of Chile, together with pictures and descriptions of 12 pollen kind typically found in this area. The results of the vegetation's collection confirm the tendency of previous studies revealing the principal altitudinal tiers of vegetation ("piso prepuneño, puneño, altoandino y subnival"). Likewise, the results of the pollen rain show an important relationship with the vegetation that produce it, making it possible to discriminate the different altitudinal tiers from its pollen signal. Thus the results can be of great interest for fossil pollen records understanding or for ecological studies.
\end{abstract}

KEYwoRDs: Palynology, pollen rain, altitudinal tiers, vegetation diversity.

\section{INTRODUCCIÓN}

Uno de los factores determinantes en la distribución de la vegetación en el Norte Grande de Chile corresponde al clima, el cual en gran medida determina la disponibilidad de agua. En efecto, el gradiente decreciente de precipitación norestesudoeste y desde mayores a menores alturas, determina una marcada zonación de los pisos de vegetación de la zona andina y preandina, entre los cuales se distinguen según su composición específica en prepuneño (predominio de matorral y cactáceas), puneño (predominio de extensiones arbustivas o tolares), altoandino (con dominio de gramíneas y plantas en cojín) y subnival (en general con especies de pequeños tamaños) (Marquet et al. 1998, Teillier 1998, Villagrán et al. 2003). Por otro lado, existe un empobrecimiento de la flora hacia el sur (Villagrán et al. 1983, Arroyo et al. 1988) caracterizándose por una baja diversidad de especies y alto grado de endemismo (Rundel et al. 1991, Moreno et al. 1994). 
Las condiciones climáticas en el Norte Grande de Chile no han sido homogéneas a lo largo del tiempo (Latorre et al. 2005). Durante el Cuaternario Tardío, la región evidenció una serie de cambios en las condiciones ambientales, relacionados con variaciones en los montos de precipitación con una tendencia general hacia condiciones más secas desde el último periodo glacial hasta la actualidad (Latorre et al. 2005, Gayo et al. 2012). Así, durante una de las fases más húmedas del Pleistoceno Tardío (14.000-9.000 años antes del presente) junto con un aumento en las precipitaciones se registró un descenso de los pisos superiores de vegetación de cerca de $1.000 \mathrm{~m}$, siguiendo el gradiente de humedad decreciente hacia bajas altitudes (Maldonado et al. 2005) y una importante densidad de ocupación humana alrededor de 10.000 años antes del presente (ej. Núñez \& Grosjean,1994, Santoro et al. 2011, Latorre et al. 2013). Durante el Holoceno medio se constató una fase aún más árida que la actual (Grosjean et al. 2001, 2003, Pueyo et al. 2011) seguida de fases más húmedas durante el Holoceno tardío (Gayo et al. 2012). Finalmente, la introducción de especies vegetales y animales exóticos en tiempos más recientes (Jaksic et al. 1997) sumadas a una fuerte actividad antrópica han terminado de modificar el paisaje (Villagrán \& Castro 2004).

Una de las maneras de reconstruir la historia ambiental de una región es a través del estudio de polen fósil. Para esto es necesario conocer la dispersión y depositación del polen actual y considerar la relación existente entre la vegetación y el polen que ésta produce (Godwin 1934a, 1934b, Erdtman 1943, Janssen
1970, D’Antoni 1991). Así los datos de composición florística, cobertura vegetal y lluvia polínica actual permiten caracterizar en términos polínicos las principales comunidades vegetales existentes en una región. Muchos autores han destacado la importancia de los estudios de lluvia polínica en la obtención de datos sobre distribución de la flora y la relación polen-vegetación (ej. Sáenz 1978, Webb 1987, Jackson 1991, Lupo 1998, Weng et al. 2004, Páez et al. 1997). Sin embargo, dicha relación o "calibración" es rara vez uno a uno ya que existen diferencias en la productividad, el transporte y la depositación del polen entre las distintas especies (Faegri \& Iversen 1989). Por otro lado, la calibración polen-vegetación representa una herramienta primordial utilizada principalmente en investigaciones sobre reconstrucción climática y/o cambios en la vegetación del pasado (Reese 2003, Rull 2006, Trivi et al. 2006, Gosling et al. 2009). Adicionalmente considerando diferentes escalas espaciales y temporales, la calibración polen-vegetación puede brindar respuestas sobre el impacto del cambio climático y/o la acción humana sobre el paisaje (Kuentz et al. 2007).

Con el objetivo de proporcionar análogos modernos que permitan calibrar los registros polínicos fósiles en el Norte Grande de Chile, este trabajo presenta las relaciones lluvia de polen-vegetación a lo largo del transecto Pozo Almonte-Salar de Huasco (PA-SH; $20^{\circ} 15^{\prime}$ S; Fig. 1). Asimismo se presenta un aporte al conocimiento de tipos polínicos de la flora actual del Norte de Chile por medio de la diagnosis palinológica de doce tipos polínicos con alta representatividad en la zona.
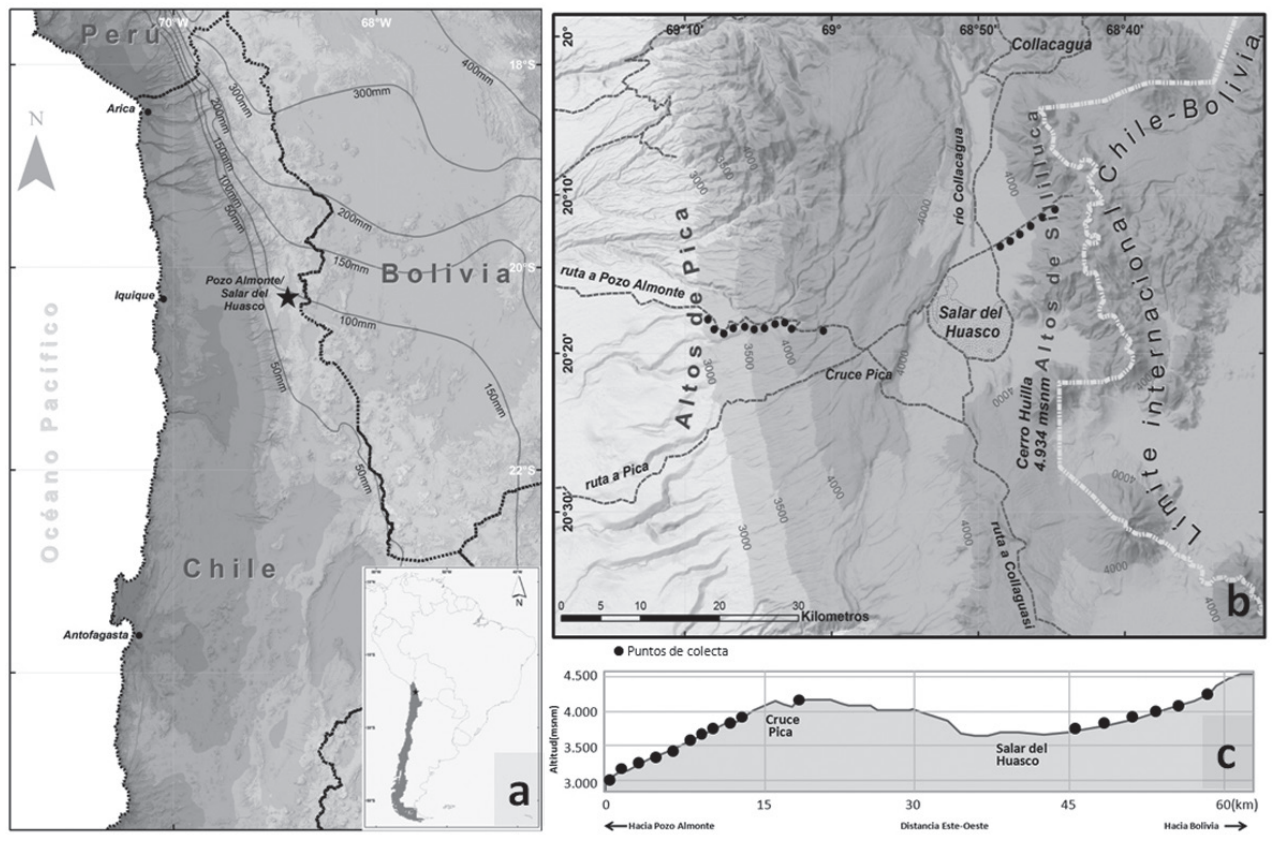

FIgURA 1. a. Ubicación del área de estudio en el Norte Grande de Chile e isoyetas de precipitación anual (datos de precipitaciones de Hijsman et al. 2005); b. puntos de muestreo (censo de vegetación y lluvia de polen) a lo largo del transecto Pozo Almonte-Salar del Huasco; c. perfil de elevación con la ubicación de los puntos de muestreo.

FigURE. 1 a. Location of the area of study in the Norte Grande area in Chile and the annual precipitation isohyets (precipitation data, Hijsman et al. 2005); b. collecting points (vegetation census and pollen rain) along the Pozo Almonte-Salar del Huasco transect; c. Elevation profile and location of the collecting points. 


\section{MATERIALES Y MÉTODOS}

El transecto en estudio se ubica a lo largo de un gradiente altitudinal Oeste-Este que se extiende desde el brazo occidental de la Cordillera de los Andes hacia el Altiplano entre la localidad de Pozo Almonte $\left(20^{\circ} 15^{\prime} \mathrm{S}\right.$; $\left.69^{\circ} 06^{\prime} \mathrm{W} ; 3.050 \mathrm{~m}\right)$ y el sector cordillerano del Salar de Huasco (20¹1'S; 68 $46^{\prime} \mathrm{W}$; $4.150 \mathrm{~m}$ ) en la cordillera de la provincia del Tamarugal (Fig.1).

VEGETACIÓN

Durante el mes de mayo del año 2009 se realizaron 17 censos de vegetación a lo largo del transecto PA-SH cada $100 \mathrm{~m}$ de altura siguiendo la metodología propuesta por McAuliffe (1990). Se contaron todos los individuos de cada taxa y se determinó su densidad mediante la asignación de una escala logarítmica dentro de una parcela de $9 \mathrm{~m}$ de radio $\left(254,5 \mathrm{~m}^{2}\right)$. Para el cálculo del porcentaje de cobertura de cada taxa se midió el diámetro de 10 individuos por taxa dentro de la parcela y se definió la mediana a la cual se asignó un valor logarítmico de cobertura que luego se extrapoló al área total muestreada para obtener la cobertura total por parcela.

Los datos de vegetación se graficaron y dividieron en grupos considerando criterios florísticos y con apoyo de un análisis de conglomerados restringido a los niveles altitudinales de cada muestreo (CONISS) utilizando TILIA 1.7.16 (Grimm 2012).

LLUVIA DE POLEN

Para el análisis de lluvia de polen se colectaron un total de 17 muestras del primer centímetro de suelo a lo largo del transecto PA-SH entre 3050 y $4150 \mathrm{~m}$, en los mismos puntos donde se realizaron los censos de vegetación.

Para la extracción de polen se procesó $1 \mathrm{~cm}^{3}$ de sedimento de cada muestra. El tratamiento consistió en: defloculación de arcillas y eliminación de ácidos húmicos con $\mathrm{KOH}$ al 10\%; remoción de los carbonatos con $\mathrm{HCl}$ al $10 \%$; remoción de silicatos con HF y posterior acetólisis para eliminar la materia orgánica (Faegri \& Iversen 1989). Se tomó una alícuota del extracto obtenido a partir de la cual se hizo un recuento superior a 300 granos de polen por muestra, utilizando microscopía óptica a 400x y 1000x de aumento.

Los granos de polen se determinaron hasta nivel de género o familia utilizando para ello muestras de referencia de la región y descripciones bibliográficas (Heusser 1971, Markgraf \& D'antoni 1978). El cálculo de los porcentajes de polen, el diagrama polínico y el análisis de conglomerados se realizaron con TILIA 1.7.16 (Grimm 2012).

\section{DesCripCión PALINOLÓGICA}

Se extrajeron las anteras de 12 especies vegetales, las que fueron tratadas con metodologías convencionales de extracción de polen (Faegri \& Iversen 1989) incluyendo $\mathrm{KOH}$ al $10 \%$ y posterior acetólisis; para el montaje y sellado de las muestras en portaobjetos se utilizó glicerina y parafina. Las muestras fueron incorporadas a la Palinoteca del Laboratorio de Paleoecología y Paleoclima del Centro de Estudios Avanzados en Zonas Áridas (CEAZA).

Para las descripciones morfológicas de los granos de polen se utilizaron 10 granos por especie y se consideraron los siguientes rasgos: forma del grano, ámbito, tamaño del grano (diámetro ecuatorial y eje polar, sin considerar la exina), aperturas $\left(\mathrm{n}^{\circ}\right.$, posición, tipo), y características de la exina (espesor y esculturas). Las fotografías de cada grano se tomaron en vista polar y ecuatorial con una cámara Canon Powershot A620 incorporada a un microscopio óptico Carl Zeiss Axiostar Plus a 400x. Finalmente las descripciones polínicas fueron apoyadas con material de la literatura (Heusser 1971, Punt et al. 2007, Herrera \& Urrego 1996).

\section{RESULTADOS}

\section{VEGETACIÓN}

La vegetación censada en el transecto estudiado está compuesta de 12 familias, 20 géneros y 25 especies, correspondiendo todas a plantas vasculares. Las familias dominantes son Asteraceae (Baccharis sp., B. tola, B. boliviensis, Parastrephia teretiuscula, P. quadrangularis, Perezia ciliosa, Senecio humillinus, Senecio sp., Werneria glaberrima), Poaceae (Deschampsia caespitosa, Festuca chrysophylla, Jarava pogonathera) y Fabaceae (Adesmia sp., A. hystrix), las cuales forman los característicos matorrales (tolares andinos) y pajonales. También es relevante la presencia de cojines de Azorella compacta (Apiaceae) y Pycnophylum macropetalum (Caryophyllaceae), además de Ephedra breana (Ephedraceae), Nototriche compacta (Malvaceae), Urbania sp. (Verbenaceae), Fabiana densa (Solanaceae), Atriplex imbricata (Chenopodiaceae) y Polylepis tarapacana (Rosaceae) (Fig.2).

A lo largo del transecto el porcentaje de cobertura total nunca excedió el 50\%, distribuyéndose entre 4 y $49 \%$ (Fig. 2). Fitogeográficamente las especies registradas son nativas y sólo Werneria glaberrima es endémica de Chile.

La vegetación ha sido dividida en cuatro grupos (I, II, III y IV) de acuerdo con el resultado del análisis de conglomerados y criterios florísticos. Estos grupos se diferencian no sólo por su composición sino además por los porcentajes de cobertura de cada uno de los taxa. Así, de menor a mayor altura, se encontraron los pisos prepuneño (Grupo I), puneño (Grupo II), altoandino (Grupo III) y subnival (Grupo IV) que son descritos, considerando exclusivamente las especies registradas dentro de cada parcela de muestreo (Fig. 2).

\section{GRUPO I (3050-3400 M)}

Este grupo se caracteriza por una cobertura total entre 5 y $20 \%$ a los 3.350 y $3.050 \mathrm{~m}$, respectivamente. Dentro de las especies Atriplex imbricata (Chenopodiaceae) tiene un dominio casi exclusivo, con porcentajes de cobertura relativa de $100 \%$ en los niveles más bajos, llegando hasta $29 \%$ en los niveles más 


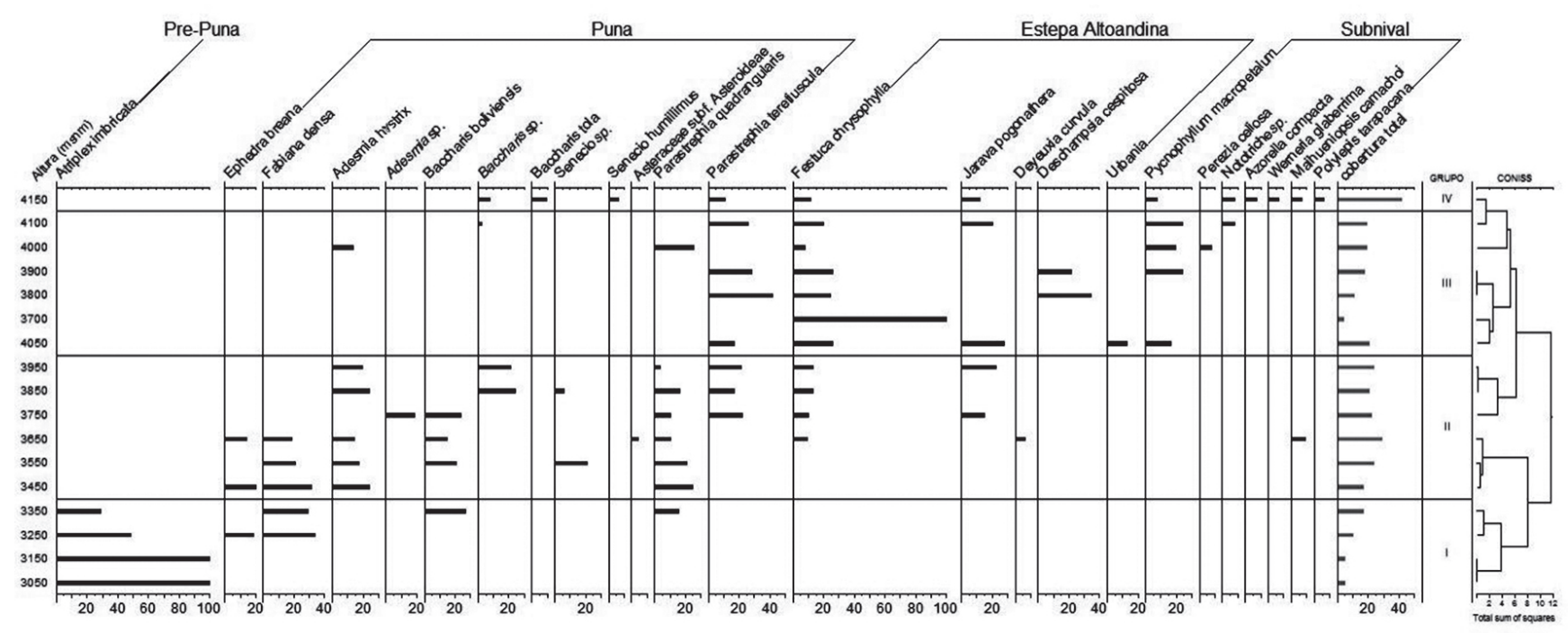

FiguRA 2. Vegetación: Diagrama de porcentajes de cobertura relativa del transecto Pozo Almonte-Salar de Huasco y dendrograma del análisis de conglomerados.

FIGURE 2. Vegetation: Diagram of percentages of relative cover from the transect Pozo Almonte-Salar de Huasco and cluster analysis.

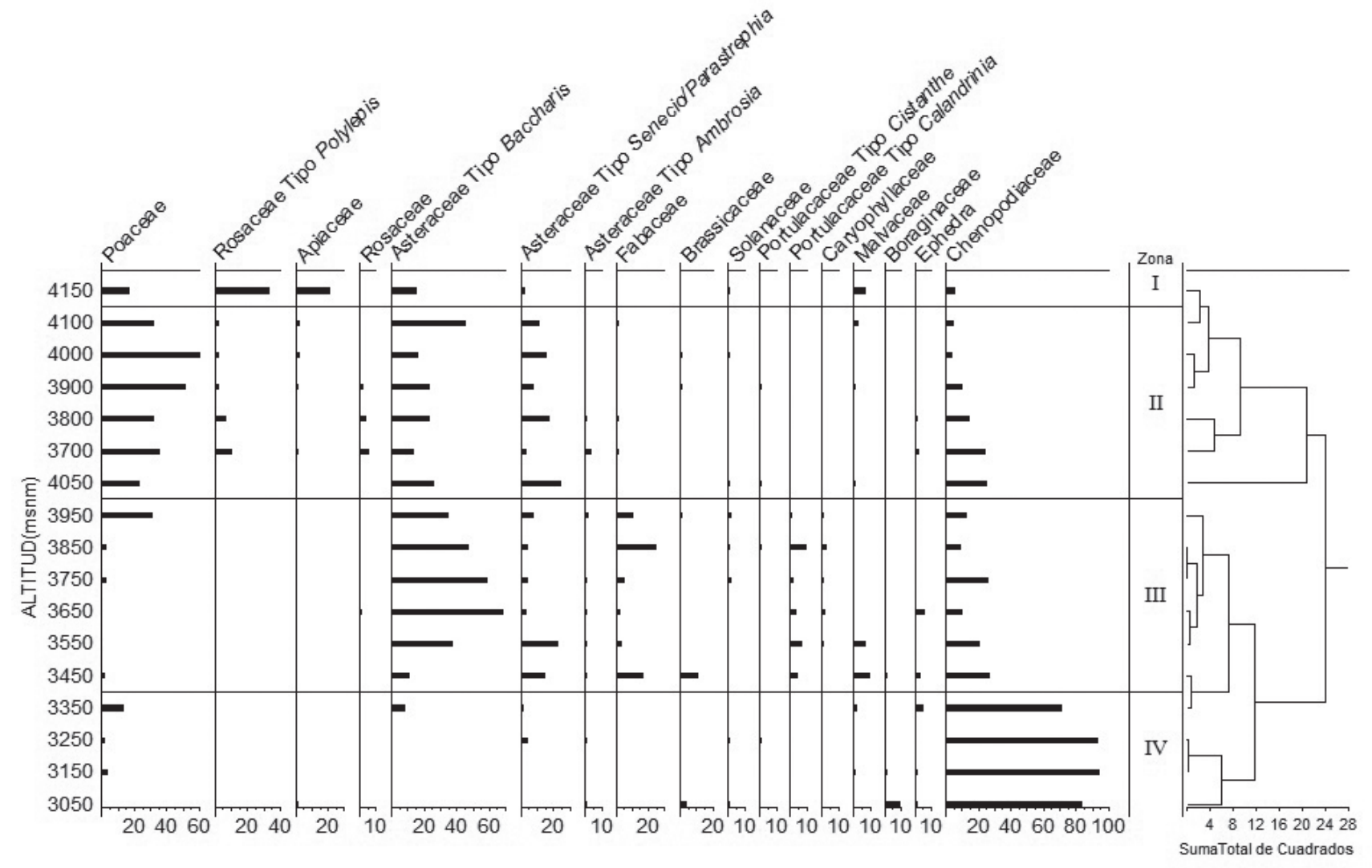

Figura 3. Polen: Diagrama de porcentajes relativos de la lluvia de polen actual del transecto Pozo Almonte-Salar de Huasco y dendrograma del análisis de conglomerados.

FIGURE 3. Pollen: Diagram of relative percentages of the current pollen rain in the transect Pozo Almonte-Salar de Huasco and cluster analysis. 
altos $(3350 \mathrm{~m})$. Las especies acompañantes en las muestras de mayor altitud de este grupo pertenecen a la familias Asteraceae (Baccharis boliviensis hasta 27\%, Parastrephia quadrangularis 16\%), Solanaceae (Fabiana densa hasta 33\%) y Ephedraceae (Ephedra breana hasta $19 \%$ ).

\section{GRUPO II (3400-4000 M)}

En este grupo la cobertura total fluctúa entre 21 y $34 \%$. Se observa una gran diversidad taxonómica con 14 especies correspondientes a 6 familias, siendo la más abundante en los niveles inferiores Solanaceae (Fabiana densa hasta 31\%), Asteraceae (Parastrephia quadrangularis hasta 24\% y Senecio sp. hasta 21\%) y Ephedraceae (Ephedra breana hasta 20\%). En los niveles superiores Poaceae (Jarava pogonathera hasta 26\%, Festuca chrysophylla hasta 13\%), Fabaceace (Adesmia hystrix hasta $24 \%$ y Adesmia sp. hasta 19\%), otras Asteraceae (Baccharis sp. hasta 24\%, B. boliviensis hasta 24\%, Parastrephia teretiuscula hasta 22\%) y porcentajes menores de Rosaceae (Polylepis tarapacana hasta 9\%), otras Poaceae (Deschampsia caespitosa hasta 7\%) y otras Asteraceae (Asteraceae subf. Asteroideae 4\%).

\section{Grupo III (4000-4100 M)}

Las muestras incluidas en este grupo se distribuyen en el extremo Este del brazo occidental de la Cordillera de los Andes, atravesando el Altiplano y extendiéndose casi hasta el límite internacional Chile-Bolivia (Fig. 1b, c). En este grupo la cobertura total fluctúa entre 4 y $24 \%$ a los $3700 \mathrm{~m}$ y a los $4.000 \mathrm{~m}$, respectivamente.

En cuanto a la diversidad vegetal se encontraron 9 especies y 5 familias. Poaceae corresponde a la familia dominante con las especies Festuca chrysophylla (hasta 100\%), Deschampsia caespitosa (hasta 35\%) y Jarava pogonathera (hasta 28\%). Hacia la parte superior la familia Asteraceae está representada por Parastrephia teretiuscula (hasta $42 \%$ ) y P. quadrangularis (hasta 26\%) y una Poaceae (Deschampsia caespitosa, hasta $35 \%$ ). Por sobre los $4000 \mathrm{~m}$ se encuentra la familia Fabaceae (Adesmia hystrix, hasta 14\%) y Caryophyllaceae con Pycnophyllum macropetalum que disminuye en abundancia desde 24 a $20 \%$ en los niveles superiores.

\section{GRupo IV (4100-4150 M)}

Este grupo corresponde a un único nivel altitudinal de muestreo a los $4150 \mathrm{~m}$. Se registraron 12 especies correspondientes a 7 familias, concentrando el mayor número de especies la familia Asteraceae. La cobertura total es de $49 \%$.

Los porcentajes de cobertura relativa muestran el dominio de Asteraceae (Parastrephia teretiuscula 26\%), Caryophyllaceae (Pycnophyllum macropetalum 24\%), Poaceae (Jarava pogonothera 20\% y Festuca chrysophylla $20 \%$ ) y porcentajes menores de Asteraceae (Baccharis tola 9\%, Baccharis sp. 7\%), Malvaceae (Nototriche sp. 8\%), Apiaceae (Azorella compacta 7\%), Rosaceae (Polylepis tarapacana 6\%) y Cactaceae (Maihueniopsis camachoi 6\%).
LLUVIA DE POLEN

En la Figura 3 se presenta el diagrama de polen en porcentaje del transecto PA-SH cuyas muestras fueron distribuidas en cuatro grupos de acuerdo a los resultados del análisis de conglomerados y criterios florísticos:

\section{Grupo I (3050-3400 M)}

Este grupo muestra el dominio de Chenopodiaceae (hasta 93\%), acompañado de bajos porcentajes de Poaceae (hasta $13 \%$ ), Boraginaceae (hasta 9\%), Brassicaceae (hasta 3\%), Asteraceae tipo Baccharis (8\%), Asteraceae tipo Senecio/ Parastrephia (hasta 4\%), Asteraceae tipo Ambrosia (1\%) y Apiaceae (1\%).

\section{GRUPO II (3400-4000 M)}

Este grupo está dominado por polen de Asteraceae tipo Baccharis (hasta 69\%) y A. tipo Senecio/Parastrephia (hasta 24\%). El polen de las familias Fabaceae (hasta 17\%), Chenopodiaceae (hasta 26\%) y Poaceae (hasta 60\%) también se encuentra en altos valores, aunque esta última sólo hacia el final de la zona. Brassicaceae (hasta 11\%) alcanza su máxima representatividad a los $3450 \mathrm{~m}$ y Portulacaceae tipo Calandrinia (hasta 9\%) a los $3850 \mathrm{~m}$.

GRUPO III (4000-4100 M)

Desde los 3900 a los $4100 \mathrm{~m}$ se registran los mayores porcentajes de polen de Poaceae alcanzando su máximo (hasta 60\%) a los $4000 \mathrm{~m}$ al igual que Asteraceae tipo Senecio/ Parastrephia (hasta 15\%). A los $4100 \mathrm{~m}$ destaca Asteraceae tipo Baccharis (hasta 45\%), Chenopodiaceae (hasta 4\%) y valores inferiores a 3\% de Malvaceae, Apiaceae, Fabaceae, Cactaceae tipo Opuntia y Ephedra. Por otro lado, el polen de Rosaceae tipo Polylepis está presente a partir de los $3700 \mathrm{~m}$ con valores también bajos (hasta $2 \%$ ).

Grupo IV (4100-4150 M)

Este grupo está dominado por polen de Rosaceae tipo Polylepis (33\%), Apiaceae (20\%), Poaceae (17\%) y Asteraceae tipo Baccharis (15\%). También se encuentra en menores porcentajes el polen de las familias Malvaceae (6\%), Chenopodiaceae (5\%) y Asteraceae tipo Senecio/ Parastrephia (2\%).

\section{DESCRIPCIÓN DE TIPOS POLÍNICOS:}

\section{Azorella compacta Phil. (Apiaceae)}

Arbusto nativo característico del piso altoandino, con crecimiento en forma de cojín que se desarrolla en hábitats rocosos por sobre $4000 \mathrm{~m}$ (Villagrán et al. 2003, Zuloaga et al. 2008).

DeSCRIPCIÓN DEL GRANo DE POLEN: Monada, prolado. Ámbito subtriangular. Diámetro ecuatorial de $11-13 \mu \mathrm{m}$ y eje polar de 17-26 $\mu \mathrm{m}$. Tricolporado, constreñido ecuatorialmente. Exina tectada de $1 \mu \mathrm{m}$ de espesor en promedio y ornamentación rugulada (Fig. 4 A.1, A. 2). 

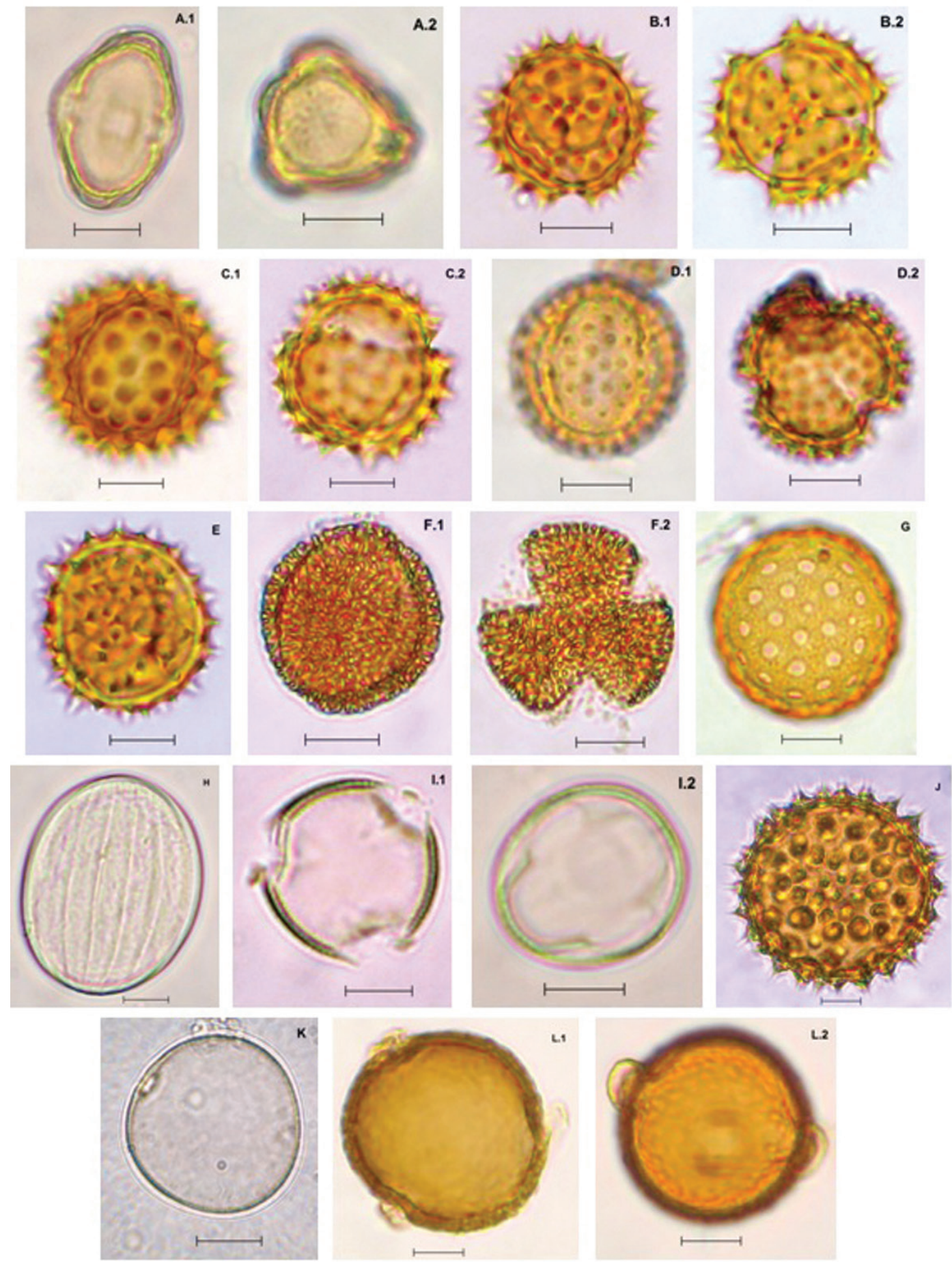

Figura 4. A.1 Azorella compacta (Vista Ecuatorial), A.2 Azorella compacta (Vista Polar); B.1 Baccharis boliviensis (V.E), B.2 Baccharis boliviensis (V.P); C.1 Parastrephia teretiuscula (V.E), C.2 Parastrephia teretiuscula (V.P); D.1 Senecio humillinus (V.E), D.2 Senecio humillinus (V.P); E Werneria glaberrima; F.1 Pycnophyllum sp. (V.E), F.2 Pycnophyllum sp (V.P); G. Atriplex imbricata; H. Ephedra breana (V.E); I.1 Adesmia sp (V.P), I.2 Adesmia sp. (V.E); J. Nototriche compacta; K. Festuca chrysophylla; L.1 Polylepis tarapacana (V.P), L.2 Polylepis tarapacana detalle poro (V.P). La barra de escala corresponde a $10 \mu \mathrm{m}$.

Figure 4. A.1 Azorella compacta (Equatorial View), A.2 Azorella compacta (Polar View); B.1 Baccharis boliviensis (E.V), B.2 Baccharis boliviensis (P.V); C.1 Parastrephia teretiuscula (E.V.), C.2 Parastrephia teretiuscula (P.V); D.1 Senecio humillinus (E.V), D.2 Senecio humillinus (P.V); E Werneria glaberrima; F.1 Pycnophyllum sp. (E.V), F.2 Pycnophyllum sp (P.V); G. Atriplex imbricata; H. Ephedra breana (E.V); I.1 Adesmia sp. (P.V), I.2 Adesmia sp. (E.V); J. Nototriche compacta; K. Festuca chrysophylla; L.1 Polylepis tarapacana (P.V), L.2 Polylepis tarapacana pore detail (P.V). Scale bar is $10 \mu \mathrm{m}$. 
2. Baccharis boliviensis (Wedd.) Cabrera (Asteraceae) Arbusto nativo característico de los tolares que se ubica entre los 3200 y 3800 m (Villagrán et al. 2003).

DesCRIPCIÓN DEL GRANO DE POLEN: Monada, subprolado a prolado-esferoidal. Ámbito circular a semiangular. Diámetro ecuatorial de $12-15 \mu \mathrm{m}$ y eje polar de 14-16 $\mu \mathrm{m}$. Tricolporado, colpos largos. Exina tectada de $0,8 \mu \mathrm{m}$ espesor en promedio y ornamentación equinada con espinas de 1,97 $\mu \mathrm{m}$ de longitud en promedio (Fig 4 B.1, B.2).

3. Parastrephia teretiuscula (Kuntze) Cabrera (Asteraceae) Arbusto nativo característico de los tolares que se encuentra entre los 3200 a $4200 \mathrm{~m}$ (Zuloaga et al. 2008).

DesCripCión Del GRANo DE polen: Monada, subprolado a prolado-esferoidal. Ámbito circular. Diámetro ecuatorial de 18-27 $\mu \mathrm{m}$ y eje polar de 21-38 $\mu \mathrm{m}$. Tricolporado. Exina tectada, de $1,0 \mu \mathrm{m}$ de espesor en promedio y ornamentación equinada con espinas de 3,0 $\mu \mathrm{m}$ de longitud promedio (Fig.4 C.1, C.2).

4. Senecio humillinus Sch. Bip. (Asteraceae)

Hierba perenne nativa con rizoma leñoso horizontal que crece sobre los $4000 \mathrm{~m}$ (Cabrera 1949)

DESCRIPCIÓN DEL GRANO DE POLEN: Monada, subprolado a prolado-esferoidal. Ámbito circular. Diámetro ecuatorial de 18-22 $\mu \mathrm{m}$ y eje polar de 20-22 $\mu \mathrm{m}$. Tricolporado. Exina tectada de $0,9 \mu \mathrm{m}$ de espesor en promedio y ornamentación equinada con espinas de 1,8 $\mu \mathrm{m}$ de longitud promedio (Fig.4 D.1, D.2).

\section{Werneria glaberrima Phil. (Asteraceae)}

Hierba perenne endémica de Chile y se encuentra entre los 4200 y 4600 m (Teillier 1998, Zuloaga et al. 2008).

DESCRIPCIÓN DEL GRANO DE POLEN: Monada, subprolado a prolado-esferoidal. Ámbito circular. Diámetro ecuatorial de 21-28 $\mu \mathrm{m}$ y eje polar de 23-29 $\mu \mathrm{m}$. Tricolporado, colpos visibles fácilmente. Exina tectada de $1 \mu \mathrm{m}$ de espesor en promedio y ornamentación equinada con espinas de 2,7 $\mu \mathrm{m}$ de longitud en promedio (Fig.4 E).

\section{Pycnophyllum sp. (Caryophyllaceae)}

Planta en cojín nativa que crece en hábitats rocosos y pampas altoandinas, por sobre $4000 \mathrm{~m}$ (Villagrán et al. 2003, Zuloaga et al. 2008).

DesCRIPCIÓN DEL GRANO De POLEN: Monada, subprodado a prolado-esferoidal. Ámbito circular. Diámetro ecuatorial de 21-27 $\mu \mathrm{m}$ y eje polar de 27-29 $\mu \mathrm{m}$. Tricolporado, colpos profundos y conspicuos. Exina intectada de 1,4 $\mu \mathrm{m}$ de espesor en promedio y con ornamentación escabrada (Fig.4 F.1,F.2).

7. Atriplex imbricata (Moq.) D.Dietr. (Chenopodiaceae) Arbusto nativo abundante en el jaral desértico y piso prepuneño, entre 2500 y $3200 \mathrm{~m}$ (Villagrán et al. 2003, Zuloaga et al. 2008).
DesCripCiÓn DEL GRANo de polen: Monada, oblado-esferoidal. Ámbito circular. Diámetro ecuatorial de 25 a $28 \mu \mathrm{m}$ y eje polar de 25 a $27 \mu \mathrm{m}$. Periporado, número de poros $\geq 35$, poros \pm circulares con membrana granular. Exina tectada de $1,82 \mu \mathrm{m}$ de espesor en promedio y ornamentación escabrada (Fig.4 G).

\section{Ephedra breana Phil. (Ephedraceae)}

Arbusto nativo que crece de preferencia en laderas secas y pedregosas de exposición norte, se encuentra desde los 500 hasta los $4300 \mathrm{~m}$ (Squeo et al. 1998, Zuloaga et al. 2008).

DESCRIPCIÓN DEL GRANO DE POLEN: Monada, subprolado a prolado-esferoidal. Ámbito circular-lobado. Diámetro ecuatorial de 27-36 $\mu \mathrm{m}$ y eje polar de 30-46 $\mu \mathrm{m}$. Inaperturado. Exina tectada de $1,4 \mu \mathrm{m}$ de espesor en promedio. Ornamentación psilada y con presencia de placas longitudinales y paralelas, separadas por surcos, las que se distribuyen uniformemente a lo largo de toda la superficie del grano (Fig.4 H).

\section{Adesmia sp. (Fabaceae)}

Subarbusto perenne endémico que se encuentra entre los 1500 a $4000 \mathrm{~m}$ (Zuloaga et al. 2008).

DESCRIPCIÓN DEL GRANO DE POLEN: Monada, subprolado a oblado-esferoidal. Ámbito circular. Diámetro ecuatorial de 20-28 $\mu \mathrm{m}$ y eje polar de 22-29 $\mu \mathrm{m}$. Tricolporado. Exina tectada de $1,0 \mu \mathrm{m}$ de espesor en promedio y ornamentación escabrada (Fig.4 I.1, I.2).

10. Nototriche compacta (A.Gray) A.W.Hill (Malvaceae)

Hierba perenne nativa que se encuentra desde los 1900 hasta los $4500 \mathrm{~m}$ (Zuloaga et al. 2008).

DESCRIPCIÓN DEL GRANO DE POLEN: Monada, prolado-esferoidal. Ámbito circular. Diámetro ecuatorial de 38-45 $\mu \mathrm{m}$ y eje polar de 38-45 $\mu \mathrm{m}$. Periporado. Exina tectada de 2,4 $\mu \mathrm{m}$ de espesor en promedio y ornamentación equinada con espinas de 5,7 $\mu \mathrm{m}$ de longitud promedio (Fig.4 J).

\section{Festuca chrysophylla Phil. (Poaceae)}

Hierba perenne nativa que se encuentra entre los 3500 y 4500 m (Zuloaga et al. 2008).

DesCRIPCIÓN DEL GRANo De POLEN: Mónada, oblado-esferoidal a prolado-esferoidal. Ámbito circular. Diámetro ecuatorial de 20 a $25 \mu \mathrm{m}$ y eje polar de 20 a $25 \mu \mathrm{m}$. Monoporado. Exina tectada de $1,1 \mu \mathrm{m}$ de espesor en promedio y ornamentación psilada (Fig.4 K).

\section{Polylepis tarapacana Phil. (Rosaceae)}

Árbol nativo que se encuentra entre los 3900 hasta los 4700 m (Zuloaga et al. 2008)

DesCRIPCIÓN DEL GRANo DE POLEN: Monada, oblado-esferoidal a prolado-esferoidal. Ámbito circular. Diámetro ecuatorial de 29 a $34 \mu \mathrm{m}$ y eje polar de 29 a $37 \mu \mathrm{m}$. Tricolporado, los colpos son cortos y los poros protruyen presentando una longitud de 
entre 4,2 y 7,8 $\mu \mathrm{m}$. Exina tectada de $1,5 \mu \mathrm{m}$ de espesor en promedio y ornamentación rugulada (Fig.4 L.1, L.2).

\section{DISCUSIÓN}

FLORA Y VEGETACIÓN

La flora registrada en este estudio está compuesta por 12 familias, 20 géneros y 25 especies. Entre las familias más representadas destacan Asteraceae (10 spp.), Poaceae (4 spp.) y Fabaceae (2 spp.), la primera formando los característicos matorrales o tolares andinos (Villagrán et al. 1983). Estos resultados concuerdan con los presentados en los trabajos de Teillier (1998) y Villagrán $(1983,2003)$ para los Andes del Norte de Chile.

Los censos de vegetación fueron divididos en cuatro grupos (Fig. 2) de acuerdo a su composición y abundancia, las que se corresponden en general a los pisos de vegetación prepuneño, puneño, altoandino y subnival, propuestos en Villagrán et al. (2003).

El grupo I, entre 3050 y $3400 \mathrm{~m}$, muestra el dominio exclusivo de Chenopodiaceae (Atriplex imbricata), pudiendo relacionarse al piso prepuneño descrito por otros autores para un amplio rango latitudinal (Villagrán et al. 1983, 2003) y asociada a bajos montos de precipitaciones, característicos de la parte inferior del gradiente de humedad del altiplano (Latorre et al. 2002, 2003, Reese \& Liu 2005). La presencia de otros taxa arbustivos como Baccharis boliviensis, Fabiana densa, Ephedra breana y Parastrephia quadrangularis hacia el final de la zona de mayor altitud dan cuenta de la transición hacia el Tolar.

En este piso, los valores de cobertura total registrados son los más bajos del transecto concordando con lo que obtuvieron para el piso prepuneño (Villagrán et al. 1981). Pese a que muchos trabajos describen el comienzo de este piso en torno a los 2500-2700 m, el inicio de la cobertura arbustiva (Baccharis boliviensis, Fabiana densa y Parastrephia quadrangularis) dentro de este piso la encontramos en torno a los $3300 \mathrm{~m}$, al igual que otros autores a esta latitud (Villagrán et al. 1981, 1999, 2003).

El grupo II, entre 3.400 y $4.000 \mathrm{~m}$, está asociado al piso puneño y su cobertura vegetal total alcanza un máximo de $34 \%$, concentrando la mayor diversidad de especies (14 especies y 6 familias), tal como lo descrito por Squeo et al (1998) para las formaciones puneñas de la zona norte de la Región de Antofagasta. Situación similar ocurre en los Andes de Arica donde este piso alcanza un máximo de 70 especies a los $3.400 \mathrm{~m}$ (Villagrán et al. 1983).

En los niveles más bajos (3400-3750 m) los arbustos Parastrephia quadrangularis, Baccharis boliviensis y Fabiana densa son los dominantes y presentan una amplia distribución altitudinal, tal como lo observado en el transecto entre Turi y Cerro León específicamente para las dos últimas especies (Villagrán et al. 1981).
El dominio de familias como Asteraceae (Baccharis, Senecio, Parastrephia, Perezia y Werneria), Fabaceae (Adesmia), Solanaceae (Fabiana) y Poaceae (Festuca, Stipa, Deschampsia), esta última en la parte superior, concuerda con otros trabajos para lugares similares del Norte de Chile (Arroyo et al. 1988, Teillier 1998, Villagrán et al. 1981, 1983) y en el caso del Salar de Ascotan (3800 m) Asteraceae y Poaceae también fueron las familias más representadas (Teillier \& Becerra 2003), determinando la transición entre el piso Puneño y el Altoandino, al igual que en nuestro estudio, donde las especies Jarava pogonathera y Festuca chrysophylla comienzan a aumentar en importancia hacia la parte alta del piso.

Los resultados obtenidos concuerdan también con otros trabajos del Sur de Perú, Weng et al. (2004) señalaron para esta altitud la presencia de Poaceae, Asteraceae y Polylepis, este último encontrado en el Norte de Chile por sobre los 4100 m (Fig. 2). En el caso de Bolivia, Ortuño et al. (2011) caracterizaron a la Puna semihúmeda a través de las familias Poaceae (Stipa y Festuca) y Asteraceae (Parastrephia y Baccharis), todas dominantes en el piso puneño del Norte de Chile. Por otra parte Kuentz et al. (2007) describieron el piso puneño sensu stricto, en el Nevado Coropuña, de manera más diversa acorde con su ubicación más ecuatorial y por lo tanto con mayor aporte de precipitaciones, compuesto por especies de las familias Apiaceae, Asteraceae, Bromeliaceae, Cactaceae, Ephedraceae, Ericaceae, Fabaceae, Rosaceae y Solanaceae.

El grupo III, ubicado entre 4000-4100 m, corresponde a la estepa altoandina e incluye la zona del altiplano, que presenta una cobertura total máxima de $25 \%$. Por otro lado, los valores mínimos de cobertura total de este grupo (4,2\%) a los $3700 \mathrm{~m}$ y su baja diversidad en este nivel, pueden estar asociados a su ubicación particular, cercanos a una estancia de pastoreo activa.

En este grupo las especies dominantes pertenecen a la familia Poaceae, especialmente Festuca chrysophylla que tiene dominio exclusivo en gran parte de esta zona. En la porción superior, Poaceae está acompañada por Asteraceae y Caryophyllaceae (Pycnophyllum macropetalum) marcando la transición hacia el piso Subnival. Villagrán et al. (1981) describieron una situación similar para el cinturón Altoandino del transecto entre Turi y Cerro León, en el cual las coberturas máximas correspondieron a gramíneas cespitosas. El aumento en importancia del cojín Pycnophyllum macropetalum en las partes más altas de la zona $(3800 \mathrm{~m})$ y la presencia de los arbustos Parastrephia teretiuscula y P. quadrangularis, resulta concordante con lo observado en otro estudio de los Andes de la I Región de Chile (Teillier 1999, Villagrán et al. 2003).

Finalmente, el grupo IV, ubicado entre los 4100-4150 m, corresponde al piso Subnival, presenta la máxima cobertura de 49\%. En este nivel las especies de Poaceae (Festuca chrysophylla y Jarava pogonathera) siguen presentes pero han disminuido su cobertura. Destaca aquí Parastrephia 
teretiuscula, la más abundante del piso junto a otras especies características de los pisos superiores, aunque con baja frecuencia, como Werneria glaberrima, Senecio humillimus, Azorella compacta y la especie arbórea Polylepis tarapacana. Esta especie que actualmente se encuentra en estado "vulnerable" de conservación (Benoit 1989), forma bosques que se distribuyen discontinuamente en laderas adyacentes al altiplano en el Norte de Chile (Villagrán et al. 1983). En otras zonas de Sudamérica, la distribución altitudinal de Polylepis tarapacana es más amplia a la presente en Chile, en Perú su presencia es registrada desde los $3200 \mathrm{~m}$ hasta altitudes mayores (Weng et al. 2004) y en Bolivia desde los $3100 \mathrm{~m}$ hasta los $4700 \mathrm{~m}$ (Ortuño et al. 2011).

Con respecto a las plantas en cojín Pycnophyllum macropetalum, Nototriche compacta y Azorella compacta crecen en sustratos rocosos y en zonas expuestas a vientos fríos (Galán de Mera et al. 2003). Esta última se encuentra por sobre los $4000 \mathrm{~m}$ y se ha propuesto que su presencia favorece la riqueza, equidad y la diversidad de las comunidades donde se encuentra permitiendo el establecimiento de otras especies a través de su efecto nodriza (Badano 2006, Luebert \& Pliscoff 2006).

En general el piso subnival y el puneño tienden a ser los más diversos (Maldonado et al. 2005, Villagrán 1983). En nuestro estudio, los valores de cobertura y diversidad concuerdan con esta tendencia, sin embargo, ambas características podrían ser aún mayores considerando que el muestreo se realizó a fines de otoño y posiblemente se excluyó la presencia de la mayoría de las plantas herbáceas.

LLUVIA DE POLEN

La lluvia de polen corresponde a un período de acumulación temporal de depositación polínica que representa la flora de un lugar, aun cuando ésta se encuentre temporalmente ausente (D'Antoni 1991). Las diferencias en los síndromes de polinización de las distintas especies de cada lugar se expresarían en la producción neta de polen y su dispersión, lo que podría explicar las diferencias entre los porcentajes de cobertura vegetal y porcentajes polínicos e incluso la ausencia de ciertos taxa en alguno de los dos set de datos.

El grupo I (Fig.3), entre 3050-3400 m, destaca por los altos porcentajes de Chenopodiaceae (93\%) y valores menores de los tipos polínicos Brassicaceae y Boraginaceae en las altitudes más bajas del transecto $(3050 \mathrm{~m})$, correspondiendo a familias características del piso prepuneño. En cuanto a la alta presencia del polen de Chenopodiaceae ésta podría explicarse por los altos valores de cobertura relativa en la vegetación (Fig. 2), sugiriendo una muy buena relación entre la lluvia de polen y la vegetación local para este piso (Fig.4).

El grupo II, entre 3400-4000 m, a lo largo del transecto existe un claro dominio del polen de Asteraceae tipo Baccharis y en las altitudes más bajas (3400 - 3550 m) también de Asteraceae tipo Senecio/Parastrephia, Brassicaceae, Chenopodiaceae y Fabaceae, esta última característica del piso puneño.
Si bien este resultado concuerda con diferentes estudios sobre lluvia polínica realizados en la puna altiplánica de Perú, Bolivia y Chile (Hansen et al. 1984, Graf 2001, Reese \& Liu 2005, Kuentz et al. 2007), éstos no diferencian entre los tipos polínicos Asteraceae tipo Senecio y Asteraceae tipo Baccharis, lo cual fue posible en este trabajo gracias a la colección de referencia que evidenció variaciones en el tamaño del grano de polen, siendo el grano de Senecio/ Parastrephia mayor a Baccharis (Fig. 4)

El grupo III, entre $4000-4100 \mathrm{~m}$, definido como piso altoandino, presenta altos valores de porcentaje de Poaceae (23 - 59\%) a lo largo de todo el grupo al igual de lo que ocurre con su cobertura vegetal. Probablemente la alta producción de polen en los pastos, acompañado de un síndrome de polinización anemófilo, favorece a su dispersión (Arroyo 1983, Reese 2003). Otros taxa presentan valores relativamente altos como las Asteraceae tipo Baccharis y Asteraceae tipo Senecio/Parastrephia, sin embargo, a esta altitud la cobertura vegetal de estas especies es baja, lo que sugiere una alta capacidad de dispersión y/o producción de éstas, situación similar sucedería con Chenopodiaceae con su alta presencia polínica en los 4 grupos (Fig. 3) pero que a nivel de vegetación sólo se ve reflejada en el grupo I (Fig. 2). Así mismo, la presencia de Rosaceae en los niveles bajos (3700-3800 m) de este grupo puede corresponder a su alta sobrerrepresentación en el polen respecto a la vegetación, ya que no hay cobertura vegetal de esta familia en los niveles bajos.

En el grupo IV, entre los 4100-4150 m, los tipos polínicos dominantes son de las familias Rosaceae tipo Polylepis (Polylepis tarapacana), que registra trazas de su polen desde los $3700 \mathrm{~m}$, Apiaceae (Azorella compacta) y Malvaceae (Nothotriche sp.), las que estarían evidenciando los elementos característicos del piso subnival, sin embargo, su señal polínica se encontraría sobrerrepresentada respecto a la vegetación, mientras que las familias menos representadas Poaceae y Asteraceae tienden a disminuir su señal polínica en relación a las zonas anteriores y en concordancia con la vegetación.

El polen de Rosaceae tipo Polylepis alcanza su máximo porcentaje (33\%) en este grupo. Este resultado concuerda con otros estudios de los Andes (Hansen et al. 1984, Kuentz et al. 2007, Ortuño et al. 2011, Sandoval 2012), los que si bien registraron otras especies de Polylepis mostraron altos porcentajes $(>30 \%)$ de granos de polen dentro de los manchones de bosque, corroborando su alta productividad polínica, sin embargo, a diferencia de los resultados encontrados por Kuentz et al. (2007), nuestros datos muestran dispersión de este taxa hasta $400 \mathrm{~m}$ más debajo de su fuente.

Para el caso de Apiaceae se ha sugerido que el polen de Azorella compacta se concentraría en ambientes de gran altitud donde crece esta especie. Fisiológicamente las flores de Azorella compacta duran poco tiempo y la liberación de secreciones del cojín atraparían parte de su propio polen, concentrando los 


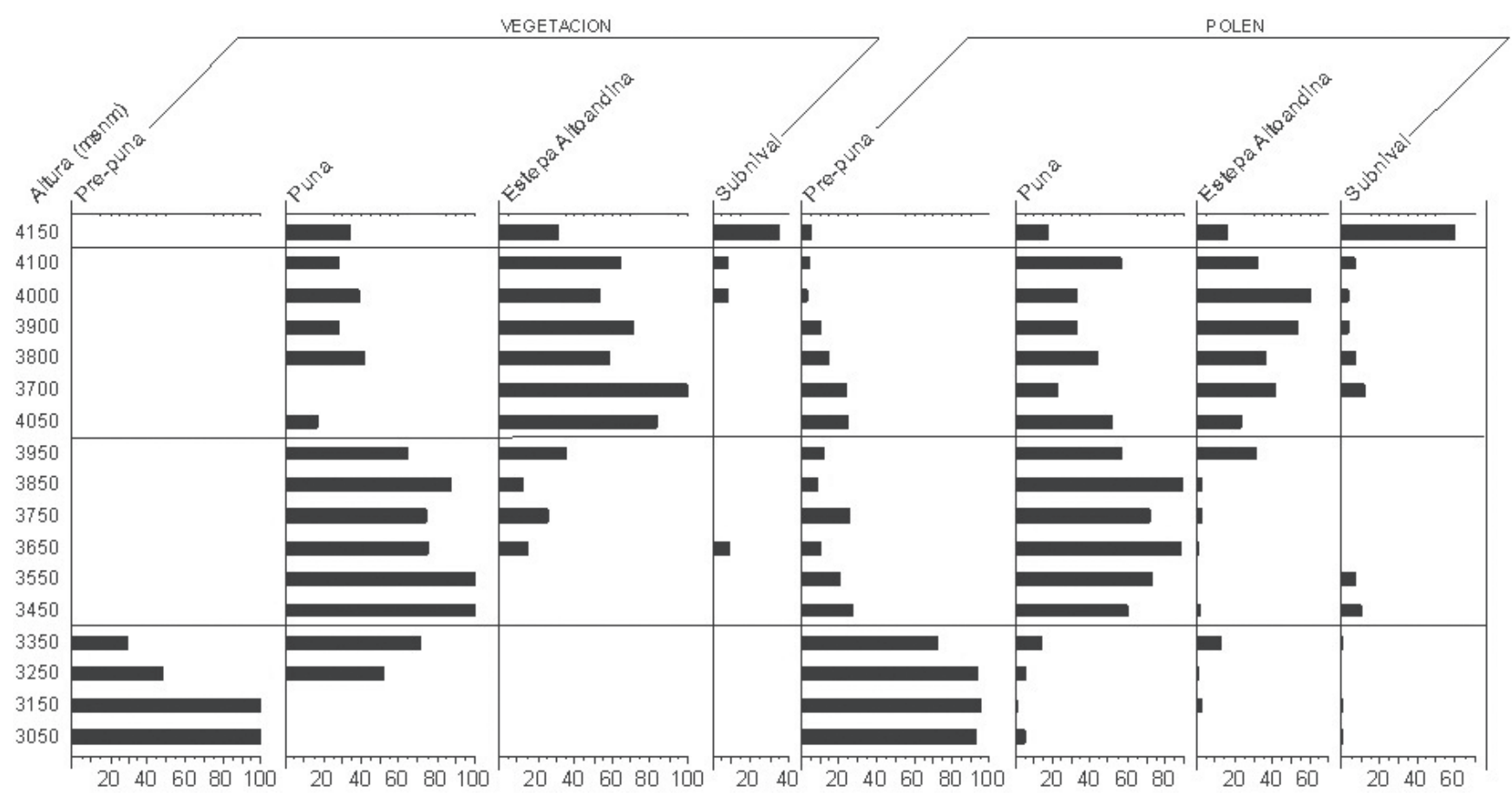

Figura 5. Gráfico integrado de vegetación y lluvia de polen de los taxa agrupados de acuerdo a los pisos de vegetación en el transecto Pozo Almonte-Salar de Huasco.

FiguRE 5. Integrative graphic of vegetation and pollen rain of taxa, it is been grouped in relation to vegetational tiers in Pozo Almonte- Salar de Huasco transect.

granos en las zonas aledañas a éste (Kuentz et al. 2007).

Así, en la mayoría de los casos las tendencias observadas en los porcentajes de cobertura relativa de vegetación muestran las mismas tendencias que los porcentajes polínicos. De igual manera, al agrupar los taxa de acuerdo a su afinidad en los pisos de vegetación (Fig. 2) se puede ver el predominio aunque no la exclusividad de estas agrupaciones a cada uno de los grupos definidos en el polen y vegetación (Fig. 5). La única excepción la constituyen los elementos de prepuna, los cuales se ubican exclusivamente en el grupo inferior. En este nivel de agrupación se puede observar también la fuerte relación entre los porcentajes relativos de vegetación y los porcentajes polínicos para cada piso de vegetación, siendo igual o más clara la señal polínica de los distintos pisos de vegetación que la señal directa de la vegetación, considerando sobre cierto nivel de representatividad polínica de cada agrupación. Así por ejemplo, una señal polínica mayor a $30 \%$ de elementos prepuneños como Chenopodiaceae dan cuenta de la presencia de este piso, y asociaciones polínicas con porcentajes mayores al 15\% entre Poaceae, Apiaceae y Rosaceae señalan la presencia del piso subnival (Fig 5). En el caso de la señal polínica de los pisos puneños y altoandino, es algo más mezclada, sin embargo el piso puneño tiende a tener un alto porcentaje de elementos del piso altoandino pero con escasez de Poaceae, mientras que el piso altoandino tiende a tener una combinación de elementos del piso puneño y de la estepa altoandina propiamente tal, al igual que en la vegetación.

En conclusión, la composición florística, censos de vegetación y lluvia de polen del transecto Pozo AlmonteSalar de Huasco muestran una congruencia en sus resultados, corroborando la relación existente entre el polen colectado y la vegetación presente (Fig. 5), siendo posible distinguir los distintos pisos de vegetación propuestos en otros trabajos para el Norte Grande de Chile (Arroyo et al. 1983, Villagrán et al. 2003).

Los pisos de vegetación y sus límites, registrados en este trabajo, tienen una clara señal en la lluvia de polen siendo posible distinguirlos exclusivamente a partir de su señal polínica, evidenciando su potencialidad como análogo moderno para estudios de polen fósil. Finalmente los tipos polínicos descritos y las diferencias morfológicas encontradas entre especies de Asteraceae tipo Senecio y Asteraceae tipo Baccharis, son un aporte al conocimiento de los granos de polen de la zona Norte de Chile y particularmente de especies con riesgos de conservación como Polylepis tarapacana y Azorella compacta (Benoit 1989). 


\section{AGRADECIMIENTOS}

Paloma Gachon, por colaborar en los muestreos de vegetación. Proyectos FONDECYT 1100916; FONDECYT 1130279; FONDECYT 3130511; FONDECYT 1080458.

\section{BIBLIOGRAFÍA}

Arroyo, M.T.K., J.J. Armesto \& C. Villagrán. 1981. Plant Phenological Patterns in the High Andean Cordillera of Central Chile. Journal of Ecology 69 (1): 205-223.

Arroyo, M.T.K., J.J. Armesto \& R. Primack. 1983. Tendencias altitudinales y latitudinales en mecanismos de polinización en la zona de los Andes templados de Sudamérica. Revista Chilena de Historia Natural 56: 159-180.

Arroyo, M.T.K., F.A. Squeo, J.J. Armesto \& C. Villagrán. 1988. Effects of aridity on plant diversity in the northern Chilean Andes: results of a natural experiment. Annals of the Missouri Botanical Garden 75: 55-78.

BADANO, E. 2006. Asociaciones de especies a plantas en cojín: sus consecuencias sobre la diversidad de especies vegetales en comunidades alto-andinas. Ecosistemas 15(1): 109-112

Benoit, I.L. 1989. Libro rojo de la flora terrestre de Chile. CONAF. $157 \mathrm{pp}$.

CABrera, A.L. 1949. El género Senecio en Chile. Lilloa 15: 27-501.

Castro, M., C. Villagrán \& M.T.K. Arroyo. 1982. Estudio etnobótanico en la precordillera y altiplano de Los Andes del norte de Chile. El Hombre y Los Ecosistemas de Montaña 133-205.

D'ANTONI, H.L. 1991. Modern pollen dispersal in southern Argentina. Bamberger Geographische Schriften 11: 209-227.

Erdtman, G. 1943. An introduction to pollen analysis. Chronica Botanica, Waltham, Massachusetts. 238 pp.

Faegri, K. \& J. Iversen. 1989. Textbook of pollen analysis. Fourth edition. John Wiley \& Sons, New York. 328 pp.

Galán de Mera, A., C. Cáceres \& A. GonzÁlez. 2003. La vegetación de la alta montaña Andina del sur del Perú. Acta Botánica Malacitana 28: 121-147.

Gayo, E.M., C. Latorre, M. Santoro, A. Maldonado \& R. De PolHoLz. 2012. Hydroclimate variability in the low-elevation Atacama Desert over the last 2500 yr Clim. Past, 8: 287-306.

Godwin, H. 1934a. Pollen analysis: an outline of the problems and potentialities of the method. I. Technique and interpretation. New Phytologist 33: 278-305.

GodwIN, H. 1934b. Pollen analysis: an outline of the problems and potentialities of the method. II. General applications of pollen analysis. New Phytologist 33: 325-358.

Gosling, W.D., J.A. Hanselman, C. Knox, B.G. Valencia \& M.B. Bush. 2009. Long term drivers of change in Polylepis woodland distribution in the central Andes. Journal of Vegetation Science 20: 1041-1052.

GRAF, K. 2001. Comparación palinológica entre el páramo y la puna de los andes sudamericanos. Asociación Paleontológica Argentina 8: 107-114.

Grimm, E. Tilia 1.7.16 Program, Illinois State Museum, Research \& Collections Center, 738 Springfield, USA, 2012.

Grosjean, M., J.F.N. Van Leeuwen, W.O. van der KnaAp, M.A. Geyh, B. Ammann, W. Tanner, B. Messerli, L. NúÑez, B.L.
Valero-Garcés \& H. Veit. 2001. A 22,000 14C year BP sediment and pollen record of climate change from Laguna Miscanti $\left(23^{\circ} \mathrm{S}\right)$, northern Chile. Global and Planetary Change 28: 35-51.

Grosjean, M., I. Cartajena, M.A. Geyh \& L. NúÑez. 2003. From proxy data to paleoclimate interpretation: the midHolocene paradox of the Atacama Desert, northern Chile. Palaeogeography, Palaeoclimatology, Palaeoecology 194: 247-258.

Hansen, B.C.S., H.E. Wright JR. \& J.P. Bradbury. 1984. Pollen studies in the Junin area, central Peruvian Andes. Geological Society of America Bulletin 95: 1454-1465.

Herrera, L.F. \& L.E. UrRego. 1996. Atlas de polen de plantas útiles y cultivadas de la Amazonia colombiana. (Reprinted from: Estudios en la Amazonia colombiana / Studies on the Colombian Amazonia, XI, 464 pp.)

Hijsmans, R.J., S.E. Cameron, J.L. Parra, P.G. Jones \& A. Jarvis. 2005. Very high resolution interpolated climate surfaces for global land areas. International Journal of Climatology. 25: 1965-1978.

Heusser, A. 1971. Pollen and spores of Chile. Modern types of the Pteridophyta, Gymnospermaea and Angiospermae. The University of Arizona Press. $167 \mathrm{pp}$.

JACKSON, S.T. 1991. Pollen representation of vegetational patterns along an elevation gradient. Journal of Vegetation Science 2: 613 - 624 .

JAKSIC, F., P. MARQuet \& Y.H. GonZÁlez. 1997. Una perspectiva ecológica sobre el uso del agua en el norte grande: La Región de Tarapacá como estudio de caso. Estudios públicos 68: 172-195.

JANSSEN, C.R. 1970. Problems in the recognition of plant communities in pollen diagrams. Vegetatio 20: 187-198.

Kuentz, A., A. Galán De Mera, M.P. Ledru \& J.P. Thouret. 2007. Phytogeographical data and modern pollen rain of the puna belt in southern Peru (Nevado Coropuna, Western Cordillera). Journal of Biogeography 32: 709-718.

Latorre, C., J.L. Betancourt, K.A. Rylander \& J. Quade. 2002. Vegetation invasions into absolute desert: A $45000 \mathrm{yr}$ rodent midden record from the Calama-Salar de Atacama basins, northern Chile (lat $\left.22^{\circ}-24^{\circ} \mathrm{S}\right)$. Geological Society of America Bulletin 114(3): 349-366.

Latorre, C., J.L. Betancourt, K.A. Rylander, J. Quade \& O. MAtтheI. 2003. A vegetation history from the arid prepuna of northern Chile $\left(22-23^{\circ} \mathrm{S}\right)$ over the last 13,500 years. Palaeogeography, Palaeoclimatology, Palaeoecology 194: 223-246.

Latorre, C., J.L. Betancourt, J.A. Rech, J. Quade, C. Holmgren, C. Placzek, A.J.C. Maldonado, M. Vuille \& K.A. Rylander. 2005. Late Quaternary history of the Atacama Desert. In: M. Smith \& P. Hesse (eds.), " $23^{\circ}$ S. The Archaeology and Environmental History of the Southern Deserts", pp. 73- 90.

Latorre, C., C.M. Santoro, P.C. Ugalde, E.M. Gayo, D. Osorio, C. Salas-Egaña, R.De Pol-Holz, D. Joly \& J.A. Rech. 2013. Late Pleistocene human occupation of the hyperarid core in the Atacama Desert, northern Chile. Quaternary Science Reviews 77: 19-30.

Luebert, F. \& P. Pliscoff. 2006. Sinopsis bioclimática y vegetacional de Chile. Editorial Universitaria. 313 pp.

LuPo, L. 1998. Estudio sobre la lluvia polínica actual y la evolución del paisaje a través de la vegetación durante el Holoceno en la cuenca del río Yavi. Borde Oriental de la Puna, Noroeste 
argentino. Tesis de doctorado, Bamberg, República Federal de Alemania. 87 pp.

MCAuliffe, J.R. 1990. A rapid method for the estimation of density and cover in desert plant communities. Journal of Vegetation Science 1(5): 653-656.

Maldonado, A., L. Betancourt, C. Latorre \& C. Villagrán. 2005. Pollen analyses from a 50000 -yr rodent midden series in the southern Atacama Desert (25 $30^{\prime}$ S). Journal of Quaternary Science 20(5): 493-507.

Markgraf, V., D'antoni, H.L.1978. Pollen flora of Argentina. University of Arizona, Tucson. 208 pp.

Marquet, P.A, F. Bozinovic, G.A. Bradshaw, C. Cornellius, H. González, J.R. Gutiérrez, E. HajeK, J.A. Lagos, F. López-Cortés, L. NúÑez, E.F. Rosello, C. Santoro, H. Samaniego, V.G. Standen, J.C. Torres-Mura \& F.M. Jaksic. 1998. Ecosistemas del desierto de Atacama y área Andina adyacente. Revista Chilena de Historia Natural 71: 593-617.

Moreno, P.I., C. Villagrán, P.A. Marquet \& L.G. Marshall. 1994. Quaternary paleobiogeography of northern and central Chile. Revista Chilena de Historia Natural 67: 487-502.

NúÑEZ, L. \& M. GRoSJEAn. 1994. Cambios ambientales pleistocenoholocénicos: Ocupación humana y uso de recursos en la Puna de Atacama (norte de Chile). Estudios atacameños 11: 7-20.

Ortuño, T., M.P. Ledru, R. Cheddadi, A. Kuentz, C. Favier \& S. BECK. 2011. Modern pollen rain, vegetation and climate in Bolivian ecoregions. Review of Paleobotany and Palynology 165: 61-74.

Páez, M.M., C. Villagrán, S. Stutz, F. Hinojosa \& R. Villa. 1997. Vegetation and pollen dispersal in the subtropical-temperate climatic transition of Chile and Argentina. Review of Palaeobotany and Palynology 96(1-2): 169-181.

Pueyo, J.J., A., Sáez, S. Giralt, B.L. Valero-Garcés, A. Moreno, R. Bao, A. Schwalb, C. Herrera, B. Klosowska \& C. Taberner. 2011. Carbonate and organic matter sedimentation and isotopic signatures in Lake Chungará, Chilean Altiplano, during the last 12.3 kyr. Palaeogeography, Palaeoclimatology, Palaeoecology 307: 339-355.

Punt, W., S. Blackmore, S.A. Nelson \& A. Lethomas. 2007. Glossary of pollen and spore terminology. Review of Palaeobotany and Palynology 143: 1-81.

Reese, C.A. 2003. Pollen dispersal and deposition in the HighCentral Andes, South America. Doctor of Philosophy. The Department of Geography and Anthropology, Louisiana State University. $132 \mathrm{pp}$.

ReEse, C. \& K. Liu. 2005. A modern pollen rain study from the central Andes region of South America. Journal of Biogeography 32: 709-718.

RuLL, V. 2006. A high mountain pollen-altitude calibration set for palaeoclimatic use in the tropical Andes. The Holocene 16: 105-117.

Rundel P.W., M.O. Dillon, B. Palma, H.A. Mooney, S.L. Gulmon \& J.R. EHLERINGER. 1991. The phytogeography and ecology of the coastal Atacama and Peruvian deserts. Aliso 13: 1-49.

Sandoval A.P. 2012. Lluvia de polen en bosques de Polylepis pepei (Rosaceae) y sus alrededores, Cordillera Oriental de los
Andes. La Paz- Bolivia. Tesis de Licenciatura en BiologíaUniversidad Mayor de San Andrés (U.M.S.A.) 95 pp.

SÁENZ, C. 1978. Introducción a la palinología y al vocabulario palinológico. H. Blume. Ediciones. Madrid. 219 pp.

Santoro, C.M., P.C. Ugalde, C. Latorre, C. Salas, D. Osorio, D. JACKSON \& E. GAYO. 2011. Ocupación humana pleistocénica en el desierto de Atacama: primeros resultados de la aplicación de un modelo predictivo de investigación interdisciplinaria. Chungara Revista de Antropología Chilena 43(1): 353-366.

Squeo, F., L.A. Cavieres, G. Arancio, J. Novoa, O. Matthei, C. Marticorena, R. Rodríguez, M.T.K. Arroyo \& M. MuÑoz. 1998. Biodiversidad de la flora vascular en la Región de Antofagasta, Chile. Revista Chilena de Historia Natural 71: 571-591.

Teillier, S. 1998. Flora y vegetación alto-andina del área de Collaguasi-Salar de Coposa, Andes del norte de Chile. Revista Chilena de Historia Natural 71: 313-329.

Teillier, S. 1999. Catálogo de las plantas vasculares del área altoandina de Salar de Coposa-Cordón Collaguasi. Chile, Región de Tarapacá (I). Chloris Chilensis. Año 2. $\mathrm{N}^{\circ} 1$. http://www.chlorischile.cl

TeILlier, S. \& P. BeCERRA. 2003. Flora y vegetación del salar de Ascotan, Andes del Norte de Chile. Gayana Botánica 60(2): 114-122.

Trivi De Mandri M.E., L. Burry \& H.L. D’Antoni. 2006. Dispersión-deposición del polen actual en Tierra del Fuego, Argentina. Revista Mexicana de Biodiversidad 77: 88-95.

Villagrán, C., J.J. Armesto \& M.T.K.Arroyo. 1981. Vegetation in high Andean transect between Turi and Cerro León in Northern Chile. Vegetatio 48: 3-16.

Villagrán, C., M.T.K. Arroyo \& C. Marticorena. 1983. Efectos de la desertización en la distribución de la flora andina de Chile. Revista Chilena de Historia Natural 56(2): 137-157.

Villagrán, C. \& V. Castro. 2004. Ciencia indígena de los Andes del norte de Chile. Editorial Universitaria, Santiago, Chile. $362 \mathrm{pp}$.

Villagrán, C., C. Castro, G. Sánchez, F. Hinojosa \& C. Latorre. 1999. La tradición altiplánica: estudio etnobotánico en los Andes de Iquique, Primera Región, Chile. Chungara Revista de Antropología Chilena 31: 86-186.

Villagrán, C., M. Romo \& V. Castro. 2003. Etnobotánica del sur de los andes de la Primera Región de Chile: un enlace entre las culturas altiplánicas y las de quebradas altas del Loa superior. Chungara Revista de Antropología Chilena 35(1): 73-124.

WeBв III, T. 1987. The appearance and disappearance of major vegetational assemblages: Long-term vegetational dynamics in eastern North. America Vegetatio 69: 177-187.

Weng, C.Y., M.B. Bush \& M.R. Silman. 2004. An analysis of modern pollen rain on an elevational gradient in southern Peru. Journal of Tropical Ecology 20: 113-124.

Zuloaga, F.O., O. Morrone \& M.J. Belgrano. 2008. Catálogo de las Plantas Vasculares del Cono Sur (Argentina, Sur de Brazil, Chile, Paraguay y Uruguay). Monographs in Systematic Botany from the Missouri Botanical Garden 107: 1-3348 pp.

Recibido: 19.02 .14

Aceptado: 19.03.15 